\title{
In -Situ Spectroscopic Investigation of Immobilized Organometallic Catalysts
}

\author{
In support of the Catalysis Science Grant entitled "Basic Principles that Govern the \\ Interaction of Organometallic Catalysts with Supports - The Science of Immobilized \\ Molecular Catalysts”
}

Final Technical Report

DOE Grant No: DE-FG02-03ER15460

DOE Program Official: Dr. Raul Miranda

Budget Period: September 15, 2003, to May 31, 2007

Principal Investigator:

Robert J. Davis

Department of Chemical Engineering

University of Virginia

Charlottesville, VA 22903-2442

Phone: 434-924-6284

FAX: 434-982-2658

e-mail: rjd4f@virginia.edu 


\section{In-Situ Spectroscopic Investigation of Immobilized Organometallic Catalysts}

Postdoctoral Researcher: Yaying Ji

Graduate Student: Surbhi Jain

Collaborators: C.W. Jones, M. Weck, P.J. Ludovice, C.D. Sherrill, all at Georgia Tech

Contact information for Robert Davis: Department of Chemical Engineering, University of Virginia, Charlottesville, VA 22904 rjd4f@virginia.edu

\section{Goals}

Immobilized organometallic catalysts, in principle, can give high rates and selectivities like homogeneous catalysts with the ease of separation enjoyed by heterogeneous catalysts. However, the science of immobilized organometallics has not been developed because the field lies at the interface between the homogeneous and heterogeneous catalysis communities. By assembling an interdisciplinary research team that can probe all aspects of immobilized organometallic catalyst design, the entire reacting system can be considered, where the transition metal complex, the complex-support interface and the properties of the support can all be considered simultaneously from both experimental and theoretical points of view. Researchers at Georgia Tech and the University of Virginia are studying the fundamental principles that can be used to understand and design future classes of immobilized organometallic catalysts.

\section{DOE Interest}

The work performed in this program elucidates fundamental principles important in the design of immobilized catalysts. These catalysts have the potential of being very active and selective while being easy to separate for the reaction medium. All of these aspects provide a substantial energy advantage in chemical processing.

\section{Publications Resulting from This Grant}

Y. Ji, S. Jain, and R.J. Davis, "Investigation of Pd Leaching from Supported Pd Catalysts during the Heck Reaction,” J. Phys. Chem. B 109 (2005) 17232-17238.

W.J. Sommer, K. Yu, J.S. Sears, Y. Ji, X. Zheng, R.J. Davis, C.D. Sherrill, C.W. Jones, and M. Weck, "Investigations into the Stability of Immobilized Pd ${ }^{\mathrm{II}}$ Pincer Complexes During Heck Catalysis,” Organometallics, 24 (2005) 4351-4361.

S. Jain, X. Zheng, C.W. Jones, M. Weck, and R.J. Davis, "Importance of Counterion Reactivity on the Deactivation of Co-Salen Catalysts in the Hydrolytic Kinetic Resolution of Epichlorohydrin,” Inorg. Chem., in press. 
S. Jain, X. Zheng, C.W. Jones, M. Weck, and R.J. Davis, "Investigation of Deactivation of Co-Salen Catalysts in the Hydrolytic Kinetic Resolution of Epichlorohydrin," submitted to the Proceedings of the Organic Reactions Catalysis Society.

\section{Presentations Acknowledging This Grant}

"Investigation of Pd Leaching during the Heck Reaction Catalyzed by Pd Supported on $\mathrm{SiO}_{2}$," Southeastern Catalysis Society, Asheville, NC, September 26-27, 2004. (with Y. Ji and S. Jain)

"Investigation of Transition Metal Leaching from Supported Pd Catalysts during the Heck Reaction,” AIChE Annual Meeting, Cincinnati, OH, Oct. 30 - Nov.4, 2005. (with S. Jain and Y. Ji)

"Heterogenization of Homogeneous Metal Complex Catalysts Degrades Catalytic Properties: Confronting the Dogma," American Chemical Society National Meeting, Atlanta, GA, March 26-30, 2006. (with K. Yu, X. Zheng, W.J. Sommer, S. Jain, M.Weck and C.W. Jones)

"Investigation of Catalyst Deactivation in the Hydrolytic Kinetic Resolution of Terminal Epoxides,” Gordon Research Conference on Catalysis, June 25-30, 2006. (poster with S. Jain)

"Effect of Counterion Reactivity on the Deactivation of Co-Salen Catalysts in the Hydrolytic Kinetic Resolution of Terminal Epoxides,” Southeastern Catalysis Society, Asheville, NC, Sept. 30- Oct.1, 2007. (with S. Jain, X. Zheng, C.W. Jones, and M. Weck)

"Role of Counterion Replacement on the Deactivation of Co-Salen Catalysts in the Hydrolytic Kinetic Resolution of Epichlorohydrin,” AICHE National Meeting, Salt Lake City, Utah, Nov. 4-9, 2007. (with S. Jain, X. Zheng, C.W. Jones, and M. Weck)

\section{Detailed Description of Results}

In the framework of the overall collaborative project with Georgia Tech, our work focused on (a) the X-ray absorption spectroscopy of an immobilized Pd-SCS-O complex (b) the mode of metal leaching from supported Pd catalysts during Heck catalysis and (c) the mode of deactivation of Jacobsen's Co-salen catalysts during the hydrolytic kinetic resolution of terminal epoxides. Catalysts containing supported Pd pincer complexes, functionalized supports containing mercapto and amine groups, and oligomeric Co-salen catalysts were synthesized at Georgia Tech and sent to the University of Virginia. Incorporation of Pd onto several different kinds of supports (silica, mercaptofunctionalized silica, zeolite Y) was performed at the University of Virginia.

\section{(a) X-ray Absorption Spectroscopy of Pd Pincer Complexes}


X-ray absorption spectroscopy was conducted on beamlines X10C and X18B at the National Synchrotron Light Source, Brookhaven National Lab, Upton, NY. The Pd K edge spectra of catalysts and reference compounds were recorded at room temperature except those collected during the Heck reaction performed at $393 \mathrm{~K}$. Spectra of samples in solvent or reaction medium were recorded in the fluorescence mode whereas other spectra were recorded in the transmission mode. For transmission measurements, ionchambers were filled with Ar to have an absorbance of 10\% in the first chamber and $80 \%$ in the second. A Pd foil (Goodfellow) was placed between the second and third ion chambers for energy calibration. At least four spectra were averaged for each sample studied in the transmission mode. For the samples examined during the Heck reaction, an in-situ cell was constructed with Teflon as body material and Kapton as the window. The reaction slurry was continuously pumped from a heated, stirred reactor vessel to the insitu cell. To improve the signal-to-noise ratio during the measurement, at least ten scans were averaged. Two data analysis methods were used in this work, specifically, fitting of a linear combination of the edge spectra and fitting of standard multi-shell EXAFS data. The data analysis was performed with the WinXAS 2.1 program. The pre-edge background was removed with a linear function and the post-edge background was subtracted with a cubic spline method. According to the Nyquist theorem, ${ }^{1}$ the number of free parameters for EXAFS curve-fitting are determined by the available data range in $k$ space and R space : Npts $=2 \Delta k^{*} \Delta \mathrm{R} / \pi+2$. Typically, 16 parameters can be determined from the data.

\section{Reference Compounds}

The XANES and EXAFS associated with the Pd K edge of different reference materials was recorded. The zero valent Pd foil revealed a distinctly different edge shape compared to the other reference compounds having Pd in a higher oxidation state. The $\mathrm{PdS}$ and $\mathrm{PdCl}_{2}$ samples have very similar XANES spectra, and the quick decay of oscillations above the peak at $\sim 24360 \mathrm{eV}$ in $\mathrm{Pd}\left(\mathrm{NH}_{3}\right)_{4} \mathrm{Cl}_{2}$ was due to the presence of light $\mathrm{N}$ atoms as the nearest neighbor backscatterers. We used the program FEFF 8.20 to calculate theoretical reference files containing the appropriate backscattering amplitudes and phase shifts for various absorber-backscatterer pairs. ${ }^{2}$ We then used the EXAFS data from the reference spectra to calibrate the reference files calculated by FEFF. The known structural parameters for each standard compound were used in the calibration process. Curve-fitting of reference data with $k^{3}$ - weighting was performed in R-space to produce a set of parameters for the calibration of theoretical FEFF reference files. Table 1 summarizes these parameters derived from curve fitting that were subsequently input to the revised theoretical FEFF reference files. ${ }^{3}$ Since a reference compound having a Pd-C first shell was not available, we used the Pd-N reference file as a substitute.

Table 1. Parameters for calibration of FEFF references derived from fits to standard compounds.

\begin{tabular}{|l|l|l|l|l|l|l|}
\hline Atom pair & $\sigma^{2}\left(\AA^{2}\right)$ & $\mathrm{S}_{0}{ }^{2}$ & $\mathrm{Vr}(\mathrm{eV})$ & $\mathrm{Vi}(\mathrm{eV})$ & $\Delta k\left(\AA^{-1}\right)$ & $\Delta \mathrm{R}(\AA)$ \\
\hline Pd-Pd & 0.00550 & 1.00 & -5.5 & 3.0 & $3.0-15.0$ & $1.5-3.2$ \\
\hline Pd-I & 0.00385 & 0.92 & 2.5 & 3.0 & $3.0-15.0$ & $1.5-3.0$ \\
\hline Pd-Cl & 0.00303 & 0.90 & 2.7 & 3.0 & $2.5-14.0$ & $1.5-2.8$ \\
\hline Pd-S & 0.00337 & 0.87 & 1.5 & 3.0 & $2.5-12.7$ & $1.5-3.0$ \\
\hline Pd-N & 0.00008 & 0.90 & 0.8 & 3.0 & $2.5-12.8$ & $1.0-2.5$ \\
\hline
\end{tabular}




\section{Influence of immobilization}

The $k^{2}$ weighted Fourier transform of the EXAFS revealed that Pd-SCS pincer complexes supported on both silica (SBA-15) and polynorbornene have light backscatterers around Pd (at $\sim 1.9 \AA$ ) associated with the pincer complex, but that a new peak was present at $\sim 2.5 \AA$ in the SBA-immobilized compound. It is reasonable to assume that this long distance may be due to the presence of some Pd in the first coordination shell, presumably from a small amount of Pd metal. Assuming the spectrum can be decomposed into contributions from the SCS pincer complex and metallic Pd, we performed a fitting analysis utilizing a linear combination of the edge spectra for SBA immobilized SCS pincer Pd. This method has been used previously to study the local structure around an absorbing atom. ${ }^{4,5}$ The XANES fitting analysis was performed in a region up to $150 \mathrm{eV}$ above the edge. The fitting parameters in Table 2 indicated that $6.5 \%$ of the Pd in SBA immobilized SCS pincer complex was metallic in nature whereas 93.3\% of the Pd was present in the SCS pincer complex. The estimated coordination number of the Pd-Pd shell was only about 0.8 .

Curve-fitting of the EXAFS data with FEFF reference files utilizing $k^{2}$ weighting in $\mathrm{R}$ space was performed on the spectra of SBA and polymer-immobilized SCS pincer complex with a fixed $\mathrm{S}_{0}{ }^{2}$ value of 0.9 . Since the number of free parameters in the fitting routine is so high (16), many are correlated, and the Nyquist theorem dictates only 16 can be independently fit, the coordination numbers and interatomic distances were fixed to a small region near the estimated values. In addition, the Debye-Waller factor was forced to be non-negative. Reasonable values of Debye-Waller factor and $\Delta \mathrm{E}_{0}$ as shown in Table 3 suggest that immobilization of the complex on the SBA support and the polymer did not cause a significant change in the atomic structure around Pd bound in the SCS pincer complex. However, curve-fitting for SBA immobilized SCS pincer Pd provided a coordination number of 1.4 and an interatomic distance of $2.71 \AA$ for Pd-Pd shell as shown in Table 3. The Pd-Pd bond length is $0.04 \AA$ shorter than that in bulk Pd, which suggests that the metallic particles must be nanometer size or less. Both fitting methods suggest that the new peak in Fourier transform of SBA-immobilized SCS pincer Pd can be assigned to the presence of small metallic Pd particles on the support, and most of Pd still remain bound in SCS pincer complex after immobilization on SBA support. It should be noted that if any $\mathrm{O}$ atoms were present on the Pd metal surface, they could not be distinguished from nearest neighbor $\mathrm{C}$ atoms associated with $\mathrm{Pd}$ in the pincer complex.

Table 2. Fitting results from linear combination of XANES $(\Delta E=24.20-24.50 \mathrm{keV})$.

\begin{tabular}{|c|c|c|c|c|c|}
\hline $\begin{array}{c}\text { Pincer } \\
\text { Compound }\end{array}$ & Measurement condition & $\begin{array}{c}\text { SCS Pincer Pd } \\
(\%)\end{array}$ & $\begin{array}{c}\text { Metallic Pd } \\
(\%)\end{array}$ & $\begin{array}{c}\mathrm{PdI}_{2} \\
(\%)\end{array}$ & $\begin{array}{c}\text { Residual factor } \\
(\%)\end{array}$ \\
\hline Poly-O-SCS & In DMF at RT & 100 & 0 & --- & 0.31 \\
\hline SBA-O-SCS & In air at RT & 93.3 & 6.5 & --- & 0.36 \\
\hline Poly-O-SCS & During Heck reaction & 85.5 & 0 & 14.5 & 0.41 \\
\hline
\end{tabular}




\begin{tabular}{|c|c|c|c|c|c|}
\hline & at 393 K & & & & \\
\hline SBA-O-SCS & $\begin{array}{c}\text { During Heck reaction } \\
\text { at 393 K }\end{array}$ & 81.7 & 2.4 & 15.7 & 0.44 \\
& & & & & \\
\hline
\end{tabular}

Table 3. Pd K edge EXAFS curve-fitting results for polymer and SBA immobilized SCS pincer compounds.

\begin{tabular}{|c|c|c|c|c|c|c|}
\hline Catalyst & $\begin{array}{l}\text { Measurement } \\
\text { condition }\end{array}$ & Scatterer & $\mathrm{CN}$ & $\mathrm{R}(\AA)$ & $\sigma^{2}\left(10^{-3} \AA\right)$ & $\Delta \mathrm{E}_{0}(\mathrm{eV})$ \\
\hline \multirow{4}{*}{ SBA-O-SCS } & \multirow{4}{*}{ In air at RT } & $S$ & 2.1 & 2.29 & 1.4 & $\begin{array}{l}-0.45 \\
\end{array}$ \\
\hline & & $\mathrm{Cl}$ & 1.0 & 2.37 & 5.0 & 4.53 \\
\hline & & $\mathrm{C}$ & 1.1 & 1.99 & 4.0 & 0.56 \\
\hline & & $\mathrm{Pd}$ & 1.4 & 2.71 & 5.4 & -4.73 \\
\hline \multirow{3}{*}{ Poly-O-SCS } & \multirow{3}{*}{ In air at RT } & $S$ & 2.3 & 2.29 & 2.1 & -3.17 \\
\hline & & $\mathrm{Cl}$ & 1.1 & 2.40 & 1.0 & 6.27 \\
\hline & & C & 1.2 & 1.99 & 3.7 & 1.73 \\
\hline \multirow{4}{*}{ Poly-O-SCS } & \multirow{4}{*}{$\begin{array}{l}\text { During Heck reaction } \\
\text { at } 393 \mathrm{~K}\end{array}$} & $S$ & 2.0 & 2.28 & 1.9 & 2.84 \\
\hline & & $\mathrm{Cl}$ & 1.1 & 2.39 & 4.0 & 6.99 \\
\hline & & $\mathrm{C}$ & 1.1 & 1.98 & 1.0 & 2.32 \\
\hline & & I & 1.2 & 2.67 & 3.6 & -1.92 \\
\hline
\end{tabular}

R-space fit, $k^{2}$ weighting, $\Delta k=2.5-12.0 \AA^{-1}, \Delta \mathrm{R}=0.8-3.2 \AA$

In-situ X-ray Absorption Spectroscopy during Heck Reaction

The influence of the solvent DMF on the structure of polymer-immobilized SCS pincer Pd was also investigated. No change in the XANES was observed between a sample in air and one dissolved in DMF. Likewise, the Fourier transform of the EXAFS region showed the same backscattering contribution in both samples. The linear combination of XANES analysis showed only the contribution from SCS pincer complex after stirring in DMF. Apparently, there is little change in the local environment around Pd with solvent addition.

To better understand the reaction mechanism of Heck catalysis, Evans et al. performed an in-situ X-ray absorption study of the Heck reaction with Pd acetate as a catalyst. ${ }^{6}$ We also performed an in-situ study during the Heck reaction catalyzed by polymer immobilized SCS Pd in DMF, with iodobenzene and butylacrylate as substrates and triethylamine as base. The Heck reaction was first carried out in a flask at $393 \mathrm{~K}$. Then the reacted solution was pumped into an in-situ cell from the reactor. A uniform suspension of catalyst in solution was achieved by recirculation between the cell and the reactor. A new feature in the Fourier transform of the EXAFS was observed at $\sim 2.5 \AA$, which could arise from the backscattering contribution of a Pd-Pd or a Pd-I shell. In order to distinguish between them, we performed a fitting of the edge spectra involving SCS 
pincer complex on polymer, metallic $\mathrm{Pd}$ and $\mathrm{PdI}_{2}$. The fitting results presented in Table 2 suggest that iodine was present in the first coordination shell of Pd. Although most of the Pd was associated with SCS pincer complex (85.5\%), a Pd-I coordination number of 0.6 was derived from the fitting procedure. Interestingly, no evidence for metallic Pd was found in the XANES. To further support this conclusion, a similar study was also performed with the SBA-immobilized SCS pincer complex, which was insoluble in DMF. In this case, the XANES fitting results also showed the presence of Pd-I in addition to a large amount of SCS pincer complex. A small amount of metallic Pd determined by the XANES fitting results may have been formed in the process of the immobilization, as discussed above. The observation of Pd-I compounds during Heck catalysis is in a good agreement with the results reported by Evans et al. ${ }^{6}$

The coordination number of Pd-I in Table 3 was about 1.2 as determined by EXAFS fitting in $\mathrm{R}$ space with $k^{2}$ weighting. The average Pd-I coordination number was slightly lower than those reported by Evans et al. (1.8 or 2.3) who studied Pd acetate as a catalyst for the Heck reaction. ${ }^{6}$ The Pd in our study remained mostly bound in the SCS pincer complex, which may be related to its higher stability during Heck catalysis compared to Pd acetate. In order to achieve a good fitting for the Pd-I contribution, the interatomic distance of Pd-I shell need be lengthened to $2.67 \AA$, which is somewhat longer than the value in $\mathrm{PdI}_{2}$. A long Pd-I bond was also observed by Evans et al. during their EXAFS study of $\left[\mathrm{Pd}_{2} \mathrm{I}_{6}\right]\left[\mathrm{NEt}_{3} \mathrm{H}\right]_{2}$ dissolved in $\mathrm{CH}_{3} \mathrm{CN}{ }^{6}$

Summary of Results from $X$-ray Absorption Spectroscopy

The X-ray absorption spectroscopic studies have provided the following information on the Pd(II) SCS-O system in support of our collaborators' results:

- The Pd species are altered under reaction conditions, with a fraction of the pincer species supported on poly(norbornene) or silica decomposing to form new species.

- Heating the immobilized species in DMF did not result in any notable change in the Pd species as determined by spectroscopy.

Furthermore, additional information obtained during this study:

- $\quad \mathrm{Pd}(\mathrm{II}) \mathrm{SCS}-\mathrm{O}$ pincer species supported on silica have a small amount of $\mathrm{Pd}(0)$ that is formed during synthesis, most likely during the immobilization step. Pd(II) SCSpincer species supported on poly(norbornene) look similar to the small molecule complex as determined by EXAFS.

- Palladium(II) iodo species are hypothesized to be the primary species formed under reaction conditions based on EXAFS and XANES analysis. ${ }^{8}$ This is consistent with our collaborators' and others works, which have found that Pd-iodo species are the primary resting state for Pd in the Heck reaction. This signal may also be enhanced in the EXAFS spectra by halide exchange under reaction conditions resulting from exchange of the $\mathrm{HI}$ formed in the reaction with the $\mathrm{Pd}-\mathrm{Cl}$ bonds in the remaining unreacted pincer complexes (only a fraction decompose) to create Pd-I bonds and liberate $\mathrm{HCl}$.

\section{(b) Investigation of Pd Leaching from Supported Pd Catalysts}


In parallel with our X-ray absorption studies, we performed a detailed evaluation of Pd leaching from supported catalysts during the Heck reaction of iodobenzene and butylacrylate in DMF solvent with triethylamine as an added base. In summary, leaching of Pd during the Heck reaction in DMF was observed from every supported Pd catalyst tested.

To clarify the causes of Pd leaching, a detailed investigation of the conditions required for Pd solubilization was performed for the three different types of catalysts (i.e. $\mathrm{Pd}-\mathrm{SiO}_{2}, \mathrm{Pd}-\mathrm{Y}$ and Pd on mercapto-functionalized silica). Each catalyst was pretreated in different solutions at $353 \mathrm{~K}$ or $373 \mathrm{~K}$ for a specified time period and then removed by filtration under hot conditions. All the reagents for the Heck reaction were subsequently added to the filtrate to initiate the homogeneous reaction at the same temperature as the pretreatment. The presence of soluble $\mathrm{Pd}$ was determined by the conversion of iodobenzene (IDB) in the homogeneous reaction of the filtrate. Adding base into DMF apparently did not leach active $\mathrm{Pd}$ from $\mathrm{Pd}-\mathrm{SiO}_{2}, \mathrm{Pd}-\mathrm{SH}-\mathrm{SiO}_{2}$ (mercapto-functionalized silica) and Pd-SH-SBA-15 (mercapto-functionalized SBA-15). However, conversion of IDB in the filtrate from unreduced, ion-exchanged Pd-Y indicates base caused some leaching of Pd from the zeolite. Pretreating catalysts in a mixture of DMF and IDB caused the filtrate from $\mathrm{Pd}-\mathrm{SiO}_{2}, \mathrm{Pd}-\mathrm{SH}-\mathrm{SiO}_{2}$ and Pd-SH-SBA-15 to be active in the conversion of IDB. Evidently all four of these catalysts leached Pd in the presence of IDB. Interestingly, IDB treatment did not cause Pd leaching from unreduced Pd-Y.

From the results of these tests, two different paths of Pd leaching were observed: treatment with IDB caused Pd leaching from all of the $\mathrm{Pd}-\mathrm{SiO}_{2}$ catalysts, with or without functionalization, and treatment with base caused leaching from unreduced Pd-Y. Since $\mathrm{Pd}-\mathrm{Y}$ contained cationic Pd in the supercages, we tested the effect of pre-reduction of Pd on the leaching path. No leaching of active Pd from a reduced Pd-Y sample (reduced in flowing $\mathrm{H}_{2}$ at $673 \mathrm{~K}$ ) was observed after treatment in the solution of base and DMF. However, a low level of activity was detected in the filtrate after pretreatment of the reduced Pd-Y in a solution of DMF along with IDB. These results are consistent with the idea that IDB leaches reduced Pd whereas base leaches cationic Pd. Recent results from our Georgia Tech collaborators indicated that leaching of Pd from immobilized Pd(II) SCS pincer complexes is caused by reaction with base, which is also consistent with our results for cationic Pd in Pd-Y. Clearly, the path of Pd leaching is correlated to the chemical state of Pd on the surface of the catalysts.

\section{(c) Investigation of Deactivation of Co-Salen Catalysts during the HKR Reaction}

The hydrolytic kinetic resolution (HKR) of terminal epoxides using Co-salen catalysts provides a convenient route to the synthesis of enantioenriched chiral compounds by selectively converting one enantiomer of the racemic mixture (with a maximum 50\% yield and $100 \%$ ee). The utilization of water as the nucleophile makes this reaction straightforward to perform at a relatively low cost. The proposed mechanism for the HKR of terminal epoxides involves the cooperativity of two Co-salen complexes, where one Co metal center activates the electrophile (epoxide) and the other Co metal center activates the nucleophile (water). Hence, the observed rate depends on the square of the 
catalyst concentration. With this mechanism in mind, researchers have developed multimeric Co-salen catalysts that exhibit activities 1-2 orders of magnitude greater than that of the monomeric Co-salen catalyst. Recently, our collaborators at Georgia Tech have prepared an oligo(cyclo-octene) supported Co-salen catalyst, with the idea that the presence of multiple Co-salen units as side chains on the oligomeric catalyst can enhance the reactivity and enantioselectivity in the HKR reaction. Most of these multimeric, homogeneous catalysts, as well as supported catalysts, are difficult to synthesize and need to be regenerated after the reaction.

We investigated possible modes of deactivation of a monomeric Co-salen catalyst during the HKR of epichlorohydrin by UV-Vis spectroscopy, X-ray absorption spectroscopy and electrospray ionization mass spectrometry combined with recycling studies without catalyst regeneration. Although an active $\mathrm{Co}(\mathrm{III})$ salen catalyst deactivated substantially after multiple cycles without regeneration, the catalyst maintained its +3 oxidation state throughout the runs. Thus, deactivation of Co-salen during HKR was not the result of Co reduction. The mass spectrum of a deactivated material showed that catalyst dimerization does not account for the loss of activity. Results from various catalyst pretreatment tests as well as from catalysts containing various counterions (acetate, tosylate, chloride and iodide) indicated that gradual addition of the Co-salen counterions to epoxide during the HKR reaction forming Co-OH salen species deactivated the catalyst. The extent of counterion addition to epoxide was influenced by the exposure time and the nucleophilicity of the counterion. An oligo(cyclo-octene) supported Co-OAc salen catalyst, which was 25 times more active than the monomeric Co-salen catalyst, was recycled multiple times with negligible deactivation. However, when the oligomeric catalyst was exposed to the HKR reactants for an extended period of time, the catalyst experienced significant loss in activity which is consistent with our findings for the molecule catalyst. This topic is the subject of our ongoing studies with our collaborators at Georgia Tech under a renewal grant from the DOE.

\section{References}

1. $\quad$ E. A. Stern, Phys. Rev. B. 1993, 48, 9825.

2. A. L. Ankudinov, B. Ravel, J. J. Rehr, S. D. Conradson, Phys. Rev. B. 1998, 58, 7565.

3. D. C. Koningsberger, B. L. Mojet, G. E. van Dorssen, D. E. Ramaker, Top. Catal. 2000, 10, 143-155.

4. J. W. Sobczak, E. Sobczak, A. Drelinkiewicz, M. Hasik, E. Wenda, J. All. and Comp. 2004, 362, 162-166.

5. T. Shido, A. Yamaguchi, Y. Inada, K. Asakura, M. Nomura, Y. Iwasawa, Top. Catal. 2002, 18, 53.

6. J. Evans, L. O’Neill, V. L. Kambhampati, G. Rayner, S. Turin, A. Genge, A. J. Dent and T. Neisius, J. Chem. Soc., Dalton Trans. 2002, 2207-2212. 